\title{
Flexor Enthesopathy of the Elbow in Three Dogs: Imaging and Surgery
}

\author{
Ciprian OBER ${ }^{1 *}$, Guenter SCHWARZ ${ }^{2}$, Ioana CRĂCIUN ${ }^{1}$, Cecilia DANCIU ${ }^{1}$, Iulia CIMPOIEȘ ${ }^{1}$ Cosmin \\ PESTTEAN $^{1}$,Iulia MELEGA ${ }^{1}$, Liviu OANA ${ }^{1}$ \\ ${ }^{1}$ Faculty of Veterinary Medicine, University of Agricultural Sciences and Veterinary Medicine Cluj-Napoca, \\ Romania,3-5 CaleaMănăștur, Cluj-Napoca, Romania \\ 2. TierklinikAniCura, Lastenstraße 2, 2020, Hollabrunn, Austria \\ *corresponding author:ciprian.ober@usamvcluj.ro
}

Bulletin UASVM Veterinary Medicine 75(2)/2018

Print ISSN 1843-5270; Electronic ISSN 1843-5378

doi:10.15835/buasvmcn-vm:2018.0012

\begin{abstract}
:
The aim of the study was to describe the radiographic and computed tomography (CT), findings in three dogs with elbow flexor enthesopathy. The study was a clinical one with client-owned dogs. In two dogs, lameness was localized to the elbow by clinical examination. Radiographic examination and CT were performed, and flexor enthesopaty was observed also in the third dog as an incidental finding. Flexorenthesopathy was diagnosed in all three dogs ( 4 joints) by combining the minimal radiographic changes with specific CT findings. Conservative and surgical treatment were performed. In all joints, any other pathology were excluded. In all three dogs, the elbow condition improved on long-term. Flexor enthesopathy at the medial epicondyle is an unrecognized condition and is a possible cause of elbow lameness in the dog.
\end{abstract}

Keywords: dog, elbow, flexor enthesopathy, radiography, computed tomography

\section{Introduction}

Flexor enthesopathy is attributed by some authors to an osseous metaplasia of the tendons of origin of the flexor muscles of the carpus and digits, traumatic avulsion of these flexor tendons, osteochondritisdissecans of the humeral trochlea, or development of an aberrant center of ossification (DeCamp et al., 2016; Grondalen, and Braut, 1976; Meyer-Lindenberg et al., 2004; Wosar et al., 1999). The term flexor enthesopathy has been proposed, (de Bakker et al., 2011), summarizing pathological changes within the flexor muscles of the carpus and digits and their attachments to the medial epicondyle, without referring to any suspected, but still unknown, etiology. Lesions of the medial epicondyle of the humerus may be comparable to the "little leaguer's elbow" or "golfer's elbow" in human beings, suggesting either a traumatic origin or an overuse lesion (de Bakker et al., 2011).
In the study of Van Ryssenet al. (2012), radiographic changes were minimal, and additional imaging techniques were necessary to demonstrate pathology in the suspected region and to exclude other pathology. The medial epicondyle shows a discrete spur and the medial epicondyle is sclerotic with a small radiolucent area (Van Ryssenet al. 2012). The medial coronoid process is well delineated and has a normal triangular shape (Van Ryssenet al. 2012). At computed tomography the medial epicondyle is sclerotic and shows a periosteal reaction (Van Ryssenet al. 2012).

Flexor enthesopathy is believed to be relatively uncommon in dogs, although an incidence of $15 \%$ was identified in a cohort study of seven litters (48 dogs) of Labrador Retrievers (Paster ER et al., 2009) and 40\% in 200 elbows (117 dogs) of dogs with lameness attributable to the elbow (de Bakker et al., 2012). This makes it a 


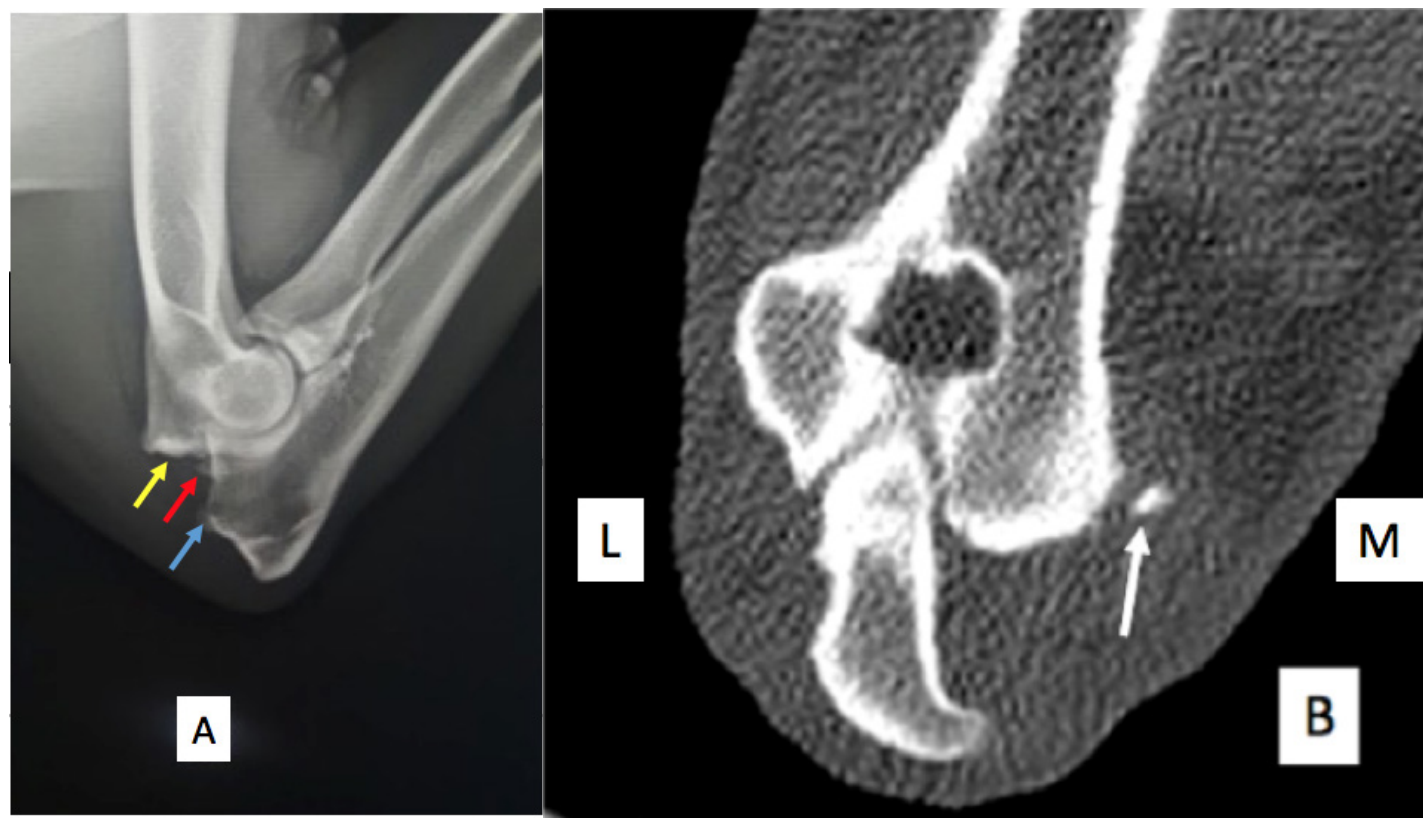

Figure 1. Latero-lateral radiographic findings in one joint affected by flexor enthesopathy. A, Rottweiler dog, twoyears-old (case 1) B, Computed tomography caudomedial slice and of a four-year-old Rottweiler (case 2) with unilateral lesion.

clinically significant differential diagnosis in any dog with thoracic limb lameness localized to the elbow. Young Labrador Retrievers seem most predisposed, followed by German Shepherd Dogs and English Setters (DeCamp et al., 2016; MeyerLindenberg et al., 2004). The term 'primary' is used, when it refers to the absence of underlying pathology in the elbow. The primary form of flexor enthesopathy can be challenging to diagnose because of minimal (or even absent) radiographic changes, which remains the first step in the diagnostic work-up. Combined with the sometimes minimal radiographic changes of medial coronoid disease makes the differentiation between medial coronoid disease and flexor enthesopathy difficult. Thus, CT and/or arthroscopy should be performed for o definitive diagnosis.

Radiography and computed tomography enabled the demonstration of bony changes in our cases. Conservative and surgical treatment improved long-term function in two dogs.

According to the authors' knowledge, this is the first report of the flexor enthesopathy of the elbow in dogs in Romania.

\section{Materials and methods}

Three dogs $(n=3)$ were recruited for the study. Two dogs were maleRottweilersaged 2 and 4 years old.The female was anRomanian Carpathian
Shepherd Dog of 6 years old.medium body weight of the dogs was $42 \mathrm{~kg}$. Clinical examination included inspection on walk and trot, assessment of the range-of-motion, joint distension, and pain response. Detailed scoring of lameness was done by assignment of grades on a scale from zero to 10 (Fuller et al., 2006). Radiographic examination was performed with the dog under anesthesia, including three standard projections of both elbows: the mediolateral flexed and extended view and $15^{\circ}$ oblique craniolateral-caudo-medial view. All dogs underwent a complete radiographic examination (Figure 1).

Two dogs had also computed tomography examination (Figure 1 and 2). The dogs were anesthetized and positioned in dorsal recumbency on the CT scanning table with the elbow joints extended approximately $135^{\circ}$. The antebrachia were positioned parallel to each other and as symmetrically as possible at the same level using a custom-made positioning device. Transverse views, perpendicular to the antebrachia, were made with a 16 slice helical CT scanner (Siemens).

\section{Results and discussions}

This paper tries to add attention to flexor enthesopathy as a differential diagnosis for elbow lameness or as an incidental finding at radiographs or computer tomography investigations. Flexor 


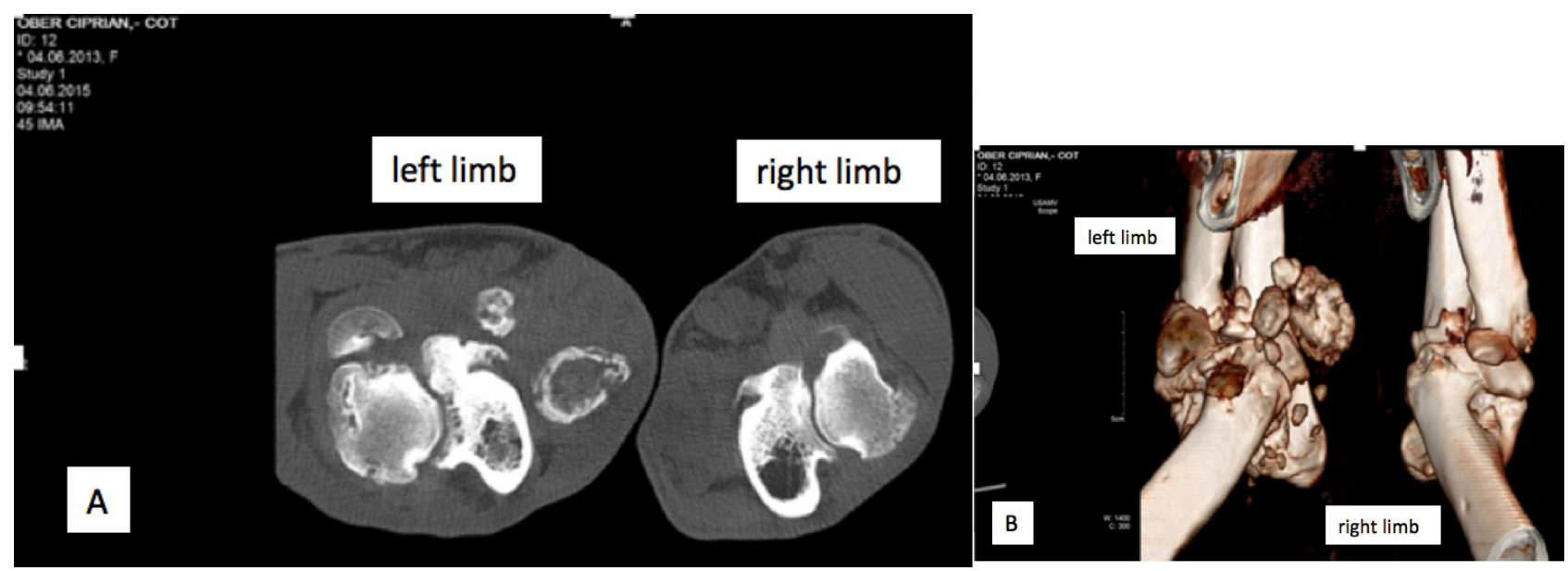

Figure 2.A, Computed tomography transverse slices and B, 3D reconstruction of a six-year-old Romanian Carpathian Shepherd Dog with bilateral lesions (case 3).

enthesopathy is still a poorly known elbow disorder and cases with obvious radiographic changes are often considered clinically unimportant (van Ryssen et al., 2012).

The imaging of three dogs with primary flexor enthesopathy showing moderate or severe changes are described. At the initial presentation, in two dogs (case 1 and 3) the combination of elbow pain and distension suggested the presumptive diagnosis of a fragmented coronoid process. The case 2 was a routine check because of a single episode of discrete lameness one month before clinical examination, according to the owner.

Latero-lateral radiographic findings in one joint affected by flexor enthesopathy in the 2-year-old Rottweiler dog (case 1) showed a combination of calcified body (red arrow), spur formation (blue arrow) and an irregular margin of the medial humeral epicondyle (yellow arrow) (Figure 1A). Computed tomography caudomedial slice and of the four-year-old Rottweiler (case 2) with unilateral lesion showed an elbow joint affected by flexor enthesopathy with a small, rounded calcified body (white arrow) medial to the joint space (Figure 1B). Computed tomography transverse slices (Figure 2A) and $3 \mathrm{D}$ reconstruction (Figure $2 \mathrm{~B}$ ) of the six-year-old Romanian Carpathian Shepherd Dog with bilateral lesions (case 3) showed the bony metaplasia within the flexor muscle, medium osteoarthritic changes and cartilaginous envelope surrounding the bony metaplasia with large mineralized tissue.
Two imaging techniques was applied in this study. Radiography and computed tomography enabled the demonstration of bony changes. The diagnosis of flexor enthesopathy was based on the presence of specific changes and exclusion of medial coronoid pathology by using two different imaging modalities.

Treatment differs with respect of the type of enthesopathy (de Bakker et al., 2011; Ciccoti and Ciccotti, 2004; Vangsness and Jobe, 1991; Gabel and Morrey, 1995) and clinical signs. Three types of management were performed for our cases: weight management with restriction of the activity for case 2, intra-articular injection of corticosteroids in case $1(0.5 \mathrm{mg} / \mathrm{kg}$ methylprednisolone $)$ and surgical removal of the affected tissue in case 3 . During surgery, the affected part of the muscles appeared to have thickened, hard and white fibrous tissue (Figure 3), located in multiple flexor muscles. The calcified tissues were connected with the surrounding tissue and joint capsule.

All dogs had complete function following treatment within three to ten weeks. According to the owners, the dogs regained full activity (case 1) and intermittent lameness in case 3 , but very good quality of life. On clinical examination the dogs (case 1 and 2) did not show any lameness, and the treated joints were not distended. In case 3, although the lameness was score one on both legs, the joints appeared distended long term after the surgery. No pain or discomfort were present, and dog had a very good quality of life, despite joint chronic distensions. 


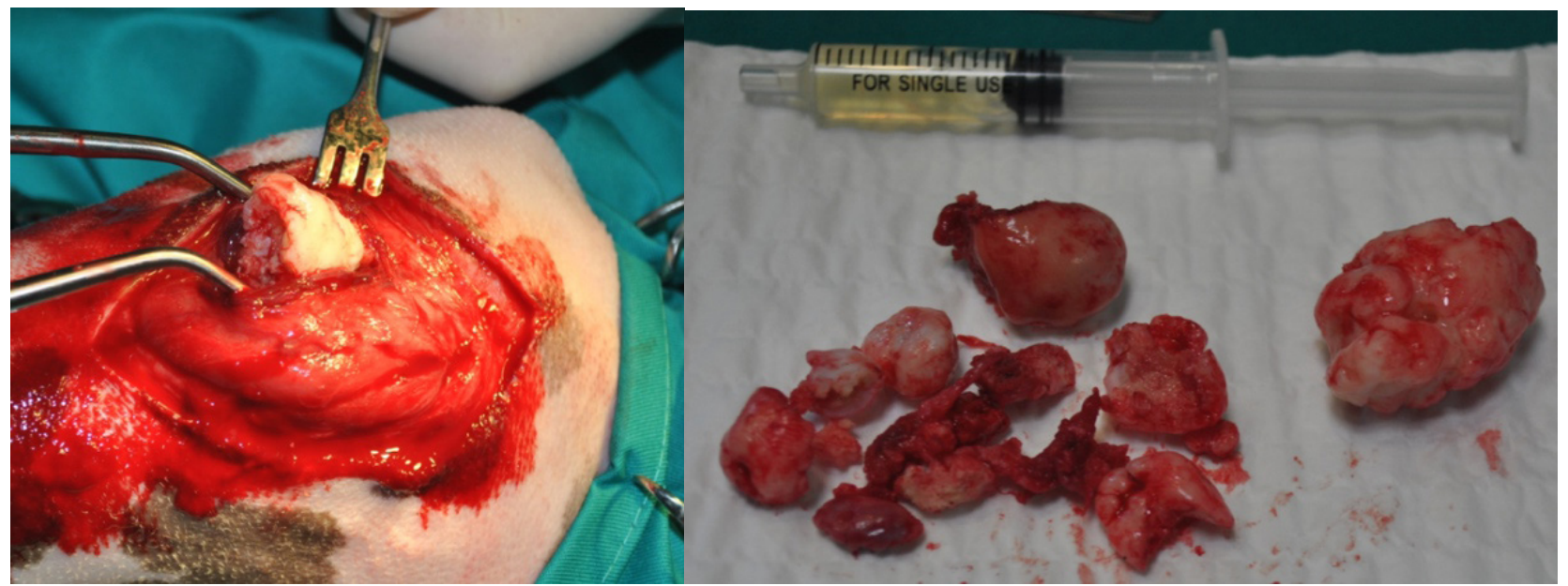

Figure 3. Intraoperative aspect of the affected tissue during surgery (case 3).

A) Thickened white/yellow proximal part of the flexor carpi ulnaris muscle.

B) Multiple large and small calcified bodies removed from the affected area

To indicate the disorder, the term 'flexor enthesopathy' adopted from human medicine (Benjamin et al., 2006) by Van Ryssen et al. (2012). In human medicine, the 'enthesis', which represents the tendon-to-bone-connection organ, has been receiving great attention lately with enthesitis or enthesopathy being considered as an important cause of locomotion problems (Van Ryssenet al., 2012). The conditions 'golfers elbow' and 'tennis elbow' are examples of this problem (Cain et al., 2003; Benjamin et al., 2006). It is unclear whether enthesopathy is the primary cause of the elbow problem or secondary to another elbow disorder in our cases.

A spur at the medial epicondyle is often considered as a sign of osteoarthritis and in that case, it is not recognized as a primary problem. In cases of 'obscure' elbow lameness (case 2), it is important to draw attention to primary flexor enthesopathy, in order to consider it in the differential diagnosis when radiographic changes are not obvious (Van Ryssen et al., 2012). Recent publications attribute obscure elbow lame- ness to fragmentation of the medial coronoid process, which is often seen as discrete lesions (Cook and Cook, 2009; Punke et al., 2009; Smith et al., 2009).

Our cases have a similar profile to dogs affected by medial compartment disease, thus, a lesion of the flexor tendons should always be considered in the differential diagnosis when 'obscure elbow lameness' is present, in order to make a correct diagnosis and treatment decision (Cook and Cook, 2009; Punke et al., 2009; Fitzpatrick and Yeadon, 2009).

This paper just recognize this elbow problem excellent presented by the team from Ghent University, Faculty of Veterinary Medicine. We agree with van Ryssen (2012) that flexor enthesopathy represents an infrequent yet important option in the differential diagnosis of elbow problems in medium and large breed dogs. Cases of primary enthesopathy may show minimal radiographic changes and hence suggest medial coronoid disease as the cause of lameness (Van Ryssenet al., 2012). A correct diagnosis of flexor enthesopathy can only be obtained by combining the radiographic findings with other imaging techniques to confirm suspected lesions of the medial epicondyle and the attaching flexors and to exclude medial coronoid disease (Van Ryssenet al., 2012).

Larger studies including more obvious cases of flexor enthesopathy with visible fragments or calcifications are being performed at Ghent University in order to define the value of each imaging modality and the clinical significance of each pathological finding.

Acknowledgments. This research did not receive any specific grant from funding agencies in the public, commercial, or not-for-profit sectors. 


\section{References}

1. Benjamin M, Toumi H, Ralphs JR et al. (2006). Where tendons and ligaments meet bone: attachment sites ('entheses') in relation to exercise and/or mech- anical load. J Anat, 208: 471-490.

2. Cain EL Jr, Dugas JR, Wolf RS, et al. (2003). Elbow injuries in throwing athletes: a current concepts review. Am J Sports Med, 31: 621-635.

3. Cook JL, Cook CR (2009). Bilateral shoulder and elbow arthroscopy in dogs with forelimb lameness: diagnostic findings and treatment outcomes. Vet Surg, 38: 224-232.

4. de Bakker E,Samoy Y, Gielen I et al. (2011). Medial humeral epicondylar lesions in the canine elbow. A review of the literature. Vet Comp OrthopTraumatol, 24:9-17.

5. de Bakker E, Saunders J, Gielen I et al. (2012). Radiographic findings of the medial humeral epicondyle in 200 canine elbow joints. Vet Comp OrthopTraumatol, 25:359-365.

6. DeCamp CE, Johnston SA, Dejardin L et al. (2016). Brinker, Piermattei, and Flo's handbook of small animal orthopaedics and fracture repair. (5th ed.). Elsevier/ Saunders: St Louis.

7. Fitzpatrick N, Yeadon R (2009). Working algorithm for treatment decision making for developmental disease of the medial compartment of the elbow in dogs. Vet Surg, 38: 285-300.

8. Fuller CJ, Bladon BM, Driver AJ et al. (2006). The intra- and inter-assessor reliability of measurement of functional outcome by lameness scoring in horses. Vet J, 171: 281286.
9. Grondalen J, Braut T (1976). Lameness in two young dogs caused by a calcified body in the joint capsule of the elbow, J Small AnimPract, 17:681-684.

10. Meyer-Lindenberg A, Heinen V, Hewicker-Trautwein M, et al. Incidence and treatment of metaplasia in the flexor tendons attached to the medial humeral condyle in the dog, TierärtztlPrax, 32:276-285.

11. Paster ER, Biery DN, Lawler DF et al. (2009). Ununited medial epicondyle of the humerus: radiographic prevalence and association with elbow osteoarthritis in a cohort of Labrador retrievers. Vet Surg, 38:169-172.

12. Punke JP, Hulse DA, Kerwin SC et al. (2009). Arthroscopic documentation of elbow cartilage pathology in dogs with clinical lameness without changes on standard radiographic projections. Vet Surg, 38: 209-212.

13. Smith TJ, Fitzpatrick N, Evans RB et al. (2009). Measurement of ulnar subtrochlear sclerosis using a percen- tage scale in labrador retrievers with minimal radiographic signs of periarticularosteophytosis. Vet Surg, 38: 199-208.

14. Van Ryssen B, de Bakker E, Beaumlin Y, Samoy YC, Van Vynckt D, Gielen I, Ducatelle R, van Bree H (2012). Primary flexor enthesopathy of the canine elbow: imaging and arthroscopic findings in eight dogs with discrete radiographic changes.Vet Comp OrthopTraumatol, 25:239-45.

15. Wosar MA, Lewis DD, Neuwirth L et al. (1999). Radiographic evaluation of elbow joints before and after surgery in dogs with possible fragmented medial coronoid process. J Am Vet Med Assoc, 214:52-58. 